\title{
Why microtubules grow and shrink
}

\author{
A new model by two physicists of the assembly of microtubules from the monomer tubulin throws an interesting \\ light on the regulation of this important and ubiquitous process.
}

THIS journal's view that molecular biologists owe it to themselves and to their subject to make molecular biology more quantitative is occasionally vindicated in the most unexpected ways. One such occasion is the appearance, in Physical Review Letters for 8 March, of an intriguing discussion of the dynamics of the growth and, conversely, the shrinkage of microtubules, the structural elements that determine the shapes of cells and which are also involved in the rearrangement of chromosomes at mitosis, for example. Although the argument is purely theoretical, it has the merit of showing up one of the most characteristic features of this system - the delicate balance there seems to be between the growth of microtubules and their shrinkage.

The assembly of a microtubule is now, in its bare bones, understood. The raw material is the protein tubulin, which can be made to polymerize to form arbitrary lengths of a microtubule. But the process is not straightforward. Nearly a decade ago, Tim Mitchison and Marc Kirschner, then both at the University of California, San Francisco, showed that, in the same in vitro preparations, some microtubules can be growing while others are shrinking (Nature 312, 232-237 \& 237-242; 1984).

Mitchison and Kirschner coined the term "dynamical instability" to describe this odd behaviour and offered as an explanation that monomers near the growing tip of a microtubule are loaded with GTP, the energy of which is required for polymerization. The result of the addition of monomers is that the GTP is converted to the diphosphate GDT. The result is that a growing polymer will tend to continue to grow, but that once it has started to shrink, it will also continue in that state. A practical consequence, which may be significant within the cell, is that some polymers will continue to grow even though most others are shrinking. More generally, dynamic instability tends to broaden the length distribution of the polymers with the passage of time.

What Marileen Dogterom and Stanislas Leibler from Princeton University and the Theoretical Physics Unit at Saclay, at Gifsur-Yvette near Paris, have now done is to clothe Mitchison and Kirschner in mathematical notation (Phys, Rev. Lett. 70, 1347; 1993), but even that provides a degree of insight into the dynamics of the growth of microtubules that has a value of its own, the inevitable crudeness of the assumptions notwithstanding.

This is how the argument goes. For the centrosomes to which microtubules are anchored during mitosis, and which are spherical structures built from protein monomers, Dogterom and Leibler suppose that one end of the microtubule is anchored to a flat surface. To simplify the geometry, they suppose that all microtubules, whatever their length, are rigid and that they grow perpendicular to the anchor-plate.

The essence of the model must be a mechanism for switching between the states of growth and of shrinkage, which is supposed to be a random process, with frequencies $f^{t}$ (for switching from shrinkage to growth) and $f^{-}$(from growth to shrinkage). Similarly, the speed with which microtubules grow and shrink are represented by $v_{+}$and $v$ respectively. The rate of growth will be influenced by the concentration of tubulin near the tip of a growing polymer, but the second is supposed to be a constant. The physical chemistry of tubulin polymerization shows that $v_{-}$may be an order of magnitude greater than the assembly speed and of the order of $20 \mu \mathrm{m}$ a second.

As it happens, the problem can even be dealt with analytically, at least if it is supposed that many microtubules grow out of the same flat anchor-plate and if, on the average, they are further apart that the characteristic diffusion length of tubulin (so that they will not compete for monomer). Then, conceptually, it is possible to define the probability density of growing and shrinking microtubule tips as a function of distance from the anchor-plate. (For example, the probability density of growing tips, $p^{+}$, at a distance $z$ from the plate will describe the number of growing tips in an infinitesimal interval $z$; thus both $p$ and $p$ are functions of $z$ and of the time, $t$.)

The upshot (assuming no competition for monomers between neighbouring polymers) is that the parameters of the problem define a sharp transition between a state in which there is unbounded growth (the average length of polymers increases with time, linearly as it happens) and one in which there is a steady state - some microtubules continue to grow, but others shrink at such a rate that the average length remains constant. The transition is defined by the condition that $v_{-} f^{-}=v_{-} f^{+}$. The statistical properties of the assembly of polymer chains can then be calculated and, in an elaboration of the model, the authors even go on to allow for competition between the growing polymers through their competition for monomer, the distribution of which is regulated by the speed with which it diffuses through the space between the growing or shrinking polymer chains.

Nobody claims that these results are more than a framework within which the general character of microtubule assembly can be discussed with a little more precision than has previously been the case. But it is plain that there are several ways in which the rate of microtubule assembly can be critically affected by small changes in the parameters of the problem, which themselves could well be determined by variations of the condition of the cell at different stages of the cell cycle.

For example, the rate at which shrinking polymers start to grow again $\left(f^{+}\right)$may well depend on the concentration of monomer, or at least on the concentration of monomer loaded with GTP, which may well itself be regulated by the activity of some phosphorylase. Or the speed of assembling polymers may be more sensitively related to the concentration of monomers than the linear relationship assumed. The plain import of the model seems to be that for microtubules to grow at all requires that exacting conditions should be satisfied. Then, small variations of any one of the parameters will send the system already in dynamic instability into some other condition altogether.

A further suggestive point arises from the authors' attempts to allow for the finite speed of monomer diffusion on the growth of a bundle of parallel microtubules. The point is that the supply of monomer near the still flat anchor-plate will have been depleted by the passage of those polymers that have already grown through the region, so that shorter polymers will have to grow with the benefit of a reduced supply of the components required for their assembly. In suitable circumstances, the resuit is that the population of microtubules growing from a fixed (and still planar) anchor-plate is split into two - those whose tips are already in the region where monomer is plentiful, and those which are left behind in the depleted region, and which are destined for repeated brief cycles of assembly and disassembly.

Nobody suggests that any of this implies that the dynamic instability of microtubules has now been made a matter of simple (or even complicated) arithmetic. That is not the function of models, at least in complicated circumstances such as those in which real-life microtubules are assembled. Rather it is that models have a heuristic value, directing attention in this case to those quantities that are likely critically to affect the behaviour of a system. That this model does excellently.

John Maddox 